Disponible en ligne : www.cahiersagricultures.fr

\title{
Entre vulnérabilité et résilience: le vécu de la pandémie de Covid-19 dans deux sociétés oasiennes du Maghreb
}

\author{
Hind Ftouhi ${ }^{1,2, *}$, Mohamed Amine Saidani ${ }^{1,3}$, Lisa Bossenbroek ${ }^{2,8}$, Meriem Farah Hamamouche ${ }^{4,5,6}$ \\ et Zakaria Kadiri ${ }^{2,7}$ \\ ${ }^{1}$ Institut Agronomique et Vétérinaire Hassan II, Rabat, Maroc \\ ${ }^{2}$ CRESC-Centre de Recherches et d'Études sur les Sociétés Contemporaines, Rabat, Maroc \\ 3 SupAgro, Montpellier, France \\ ${ }^{4}$ CIRAD, UMR G-EAU, F-34398 Montpellier, France \\ ${ }^{5}$ G-EAU, Univ Montpellier, Montpellier, France \\ ${ }^{6}$ Bureau de Recherche et de Développement Agricole (BRDA), Paris, France \\ ${ }^{7}$ Hassan II University of Casablanca, Faculté des Lettres et des Sciences Humaines, Ain Chock, Maroc \\ ${ }^{8}$ IES Landau, Institute for Environmental Sciences, University of Koblenz-Landau, Landau, Germany
}

\begin{abstract}
Résumé - À partir de la mi-mars 2020, les gouvernements algérien et marocain ont instauré un confinement pour lutter contre la propagation de la pandémie de Covid-19. Cela s'est traduit par la fermeture des marchés locaux et des restrictions de mobilité. Le traitement médiatique et scientifique de l'impact de la pandémie a porté principalement sur les zones urbaines, laissant de côté les expériences des ruraux alors même que les mesures restrictives sont arrivées en pleine campagne agricole. Cet article vise à combler partiellement cette lacune en analysant comment les petits agriculteurs, femmes et hommes des oasis et des extensions oasiennes, les jeunes producteurs et les ouvriers de deux régions oasiennes du Maghreb, ont vécu ces nouveaux défis. Pour ce faire, nous nous appuyons sur 150 entretiens téléphoniques menés avec des acteurs ruraux dans les vallées du Drâa (Maroc) et du M’zab (Algérie). En mettant leurs expériences au cœur de notre analyse, nous montrons comment la crise sanitaire a limité la capacité de la majorité des petits agriculteurs à écouler leur production agricole, et comment elle a mis à l'épreuve la pluriactivité des familles oasiennes, les rendant ainsi plus vulnérables. Puis, nous décrivons comment ces acteurs ont développé différentes pratiques de résilience, individuelles et collectives, comme la mise en place d'un marché du travail virtuel pour remplacer les traditionnels moquefs (places de recrutement des ouvriers), la transition vers des pratiques agroécologiques, la réinvention de la solidarité et de l'action collective.
\end{abstract}

Mots clés : Covid-19 / oasis / résilience / vulnérabilité / Maghreb

Abstract - Between vulnerability and resilience: the experience of the Covid-19 pandemic in two oasis societies in the Maghreb region. From mid-March 2020 onwards, the Algerian and Moroccan governments instituted a lockdown to prevent the spread of the Covid-19 pandemic, resulting in the closure of local markets and mobility restrictions. Even though these measures were implemented halfway through the agricultural year, the media and scientific coverage of the impact of the pandemic focused mainly on urban areas, leaving aside the experiences of rural people. This article aims to partially fill this gap by analysing how smallholder farmers, women and men, young producers, and workers in two oasis regions in the Maghreb, experienced these new challenges. To do so, we rely on 150 phone interviews conducted with rural actors in the Drâa Valley (Morocco) and the M'zab Valley (Algeria). By putting their experiences at the heart of our analysis, we show how the health crisis has put constraints on the ability of the majority of smallholder farmers to sell their products and how it has put a strain on the various activities of oasis families, making them more vulnerable. Then, we illustrate how these actors developed different individual

\footnotetext{
*Auteur de correspondance : hindftouhi@gmail.com
} 
and collective resilience practices, through the establishment of a virtual labor market to replace the traditional moquefs (places of recruitment of workers), the adoption of agroecological practices, and the reinvention of solidarity and collective action.

Keywords: Covid-19 / oasis / resilience / vulnerability / Maghreb

\section{Introduction}

À partir de la mi-mars 2020, les gouvernements algérien et marocain ont instauré un confinement pour lutter contre la propagation de la pandémie de Covid-19. Cela s'est traduit par la fermeture des souks (marchés locaux), des restrictions de mobilité (nationales et internationales), une complexité des procédures pour se déplacer à l'intérieur des régions et entre régions, et l'interdiction des rassemblements. En milieu rural, ces mesures sont arrivées en pleine campagne de récolte et de commercialisation des pastèques (avril) et des céréales (avrilmai). Toutefois, l'intérêt médiatique et scientifique a principalement porté sur les expériences des citadins, tandis que les travaux sur le vécu des ruraux sont restés rares (Mahdi, 2020; Hamamouche et Saidani, 2020 ; Kadiri, 2020 ; Bossenbroek et Ftouhi, 2021 ; Leonardelli et al., 2021). Pourtant, la population rurale constitue $37 \%$ de la population marocaine et $27 \%$ de la population algérienne (Banque Mondiale, 2019); l'agriculture joue un rôle économique capital, paradoxe d'un secteur qui offre beaucoup d'emplois (et garantit la sécurité alimentaire) tout en étant caractérisé par le sous-emploi (Amichi et al., 2015). Enfin, dans les deux pays, les populations rurales sont vulnérables et vivent dans des conditions socio-économiques précaires, comme en témoignent les taux élevés de pauvreté recensés par les deux gouvernements.

À la lumière de ces éléments et de nos travaux antérieurs avec différents acteurs ruraux dans deux régions oasiennes d'Algérie et du Maroc, nous nous sommes demandé comment réagissaient ces acteurs face aux contraintes liées à la pandémie. Comment les petits agriculteurs ont-ils commercialisé leurs produits alors que les souks étaient fermés? Comment les ouvriers agricoles ont-ils pu se rendre dans les exploitations agricoles? Car si les urbains connaissent le télétravail, les ruraux ne peuvent « télé-gérer » leurs champs ou «télé-alimenter» leurs troupeaux (Kadiri, 2020). En d'autres termes: quels ont été les impacts de la pandémie sur les pratiques agricoles et comment les acteurs ruraux ont-ils surmonté ces défis?

Dans cet article, nous cherchons à répondre à ces questions tout en mettant au cœur de notre analyse le vécu des petits agriculteurs, femmes et hommes des oasis traditionnelles et de leurs extensions, des jeunes producteurs et des ouvriers, dévoilant ainsi leur vulnérabilité, leur résilience et leur ingéniosité face à des situations critiques. Nous considérons la vulnérabilité à travers sa dimension sociale et l'exposition des groupes et des individus à des situations de stress externes qui se traduisent par la perturbation des moyens de subsistance et la perte de sécurité (Adger, 2000). Ces tensions sont souvent liées à la situation économique et sociale sous-jacente (manque de revenus et de ressources) (Amichi et al., 2015). Cependant, ces acteurs ruraux sont résilients et ne sont pas démunis de toute capacité de réaction. Par résilience, nous entendons la capacité des communautés à faire face aux chocs et aux menaces sociales, politiques et environnementales externes (Keck et Sakdapolrak, 2013), mais aussi «à s'y préparer, voire à les éviter et s'y adapter sur le long terme» (Lallau et al., 2018). Bien que ce concept soit utile pour apprécier l'ingéniosité des acteurs locaux et/ou des communautés, il est important de rester critique quant à son utilisation. Mal défini, ce concept peut avoir une connotation néolibérale, selon laquelle chacun est capable de s'en sortir par lui-même, de se construire un avenir en dépit des difficultés, négligeant ainsi le poids des structures (Lallau, 2019). D'autre part, le concept aurait aussi un côté conservateur dans la mesure où il viserait plutôt un retour au statu quo ante (Lallau et al., 2018). Nous considérons ici plutôt que les menaces extérieures peuvent être perçues comme des opportunités pour changer et innover. À travers cette compréhension conceptuelle, nous nous intéressons à la vulnérabilité et aux pratiques de résilience des différents acteurs ruraux et à leur (in)capacité à faire face aux mesures instaurées pour lutter contre la pandémie.

\section{Zones d'étude}

L'étude s'est déroulée dans deux régions sahariennes du Maghreb, la vallée du M'zab en Algérie et la vallée du Drâa au Maroc. L'intérêt d'une approche comparative renvoie aux similitudes des contextes oasiens et des évolutions agraires de ces deux cas d'étude. La vallée du M'zab comprend cinq oasis qui s'étendent sur une superficie de 1500 ha (Fig. 1). L'agriculture y repose sur un système d'irrigation consistant à dériver les eaux de crue des principaux oueds provenant de l'Atlas saharien pour l'irrigation et la recharge de la nappe phréatique. La vallée du Drâa, quant à elle, est caractérisée par une ceinture de $\pm 200 \mathrm{~km}$ de six oasis (Fig. 1). Ici, l'eau de surface régulée par le barrage El Mansour Eddahbi est essentielle. Pour irriguer, les agriculteurs comptent sur les lâchers de barrage et sur les eaux souterraines, elles-mêmes tributaires des écoulements de surface de l'Oued Drâa.

Dans les deux vallées, l'agriculture et l'élevage sont essentiels pour les habitants. L'agriculture est caractérisée par un système de production stratifié associant palmier dattier, arbres fruitiers et cultures annuelles. Lors des dernières décennies, l'accès au foncier et aux eaux souterraines, couplé aux encouragements des pouvoirs publics par l'octroi de subventions, ont contribué à l'émergence d'extensions agricoles en lisière des anciennes oasis. Cela a également conduit à une sédentarisation des éleveurs qui pratiquent désormais l'élevage moins qu'avant. Une production laitière existe principalement pour la consommation domestique. En plus des cultures vivrières, les agriculteurs locaux et des investisseurs nationaux ont introduit des cultures à haute valeur ajoutée destinées aux marchés nationaux et internationaux, comme les dattes Deglet Nour dans le M'zab et les pastèques dans le Drâa. Dans les deux zones, une activité touristique existe et l'exode est très important. Ces zones 


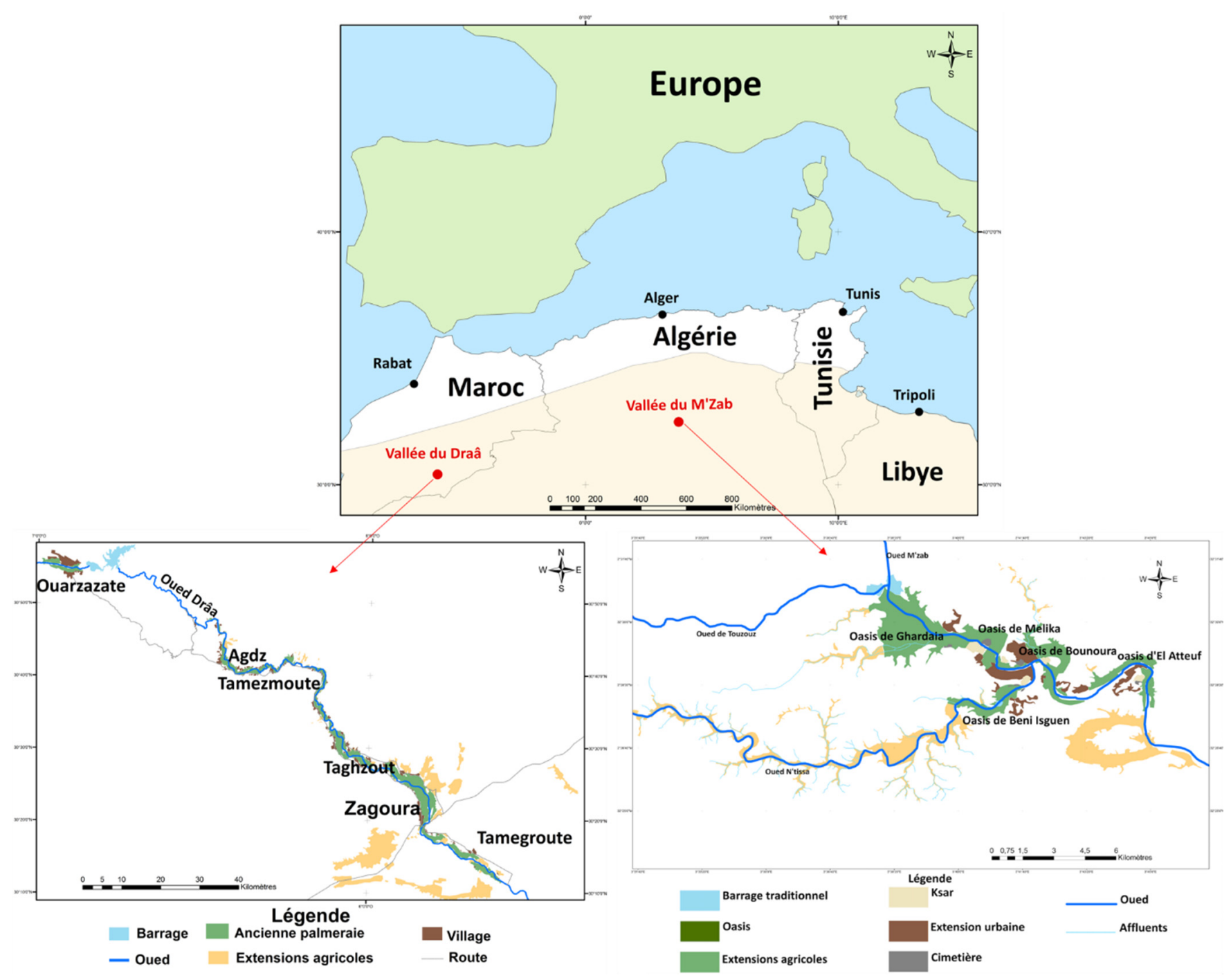

Fig. 1. Zones d'étude.

Fig. 1. Study areas.

connaissent aussi une dynamique associative importante qui a servi de base à des actions collectives pour faire face aux impacts de la pandémie.

\section{Méthodologie: un travail de terrain à distance}

Cette étude se base sur nos travaux antérieurs à la pandémie avec les communautés oasiennes pour des recherches sur les dynamiques agraires et rurales. Au moment de l'instauration du confinement, nous avons maintenu le contact avec différents acteurs ruraux. Cependant, compte tenu du confinement et des restrictions de mobilité, nous avons dû opter pour un travail de terrain à distance. Ainsi, entre avril et décembre 2020, 150 entretiens téléphoniques semi-directifs (70 dans le M'zab et 80 dans le Drâa) ont été menés auprès des petits agriculteurs, jeunes producteurs de pastèques du Drâa et de dattes du M'zab, des ouvriers, de certaines familles et de femmes des oasis traditionnelles et de leurs extensions. D'autres entretiens ont été mené avec des ouvriers agricoles, des membres d'associations et de coopératives et des représentants étatiques. Les entretiens téléphoniques ont été complétés par des échanges sur WhatsApp sous forme de photos, vidéos et messages vocaux. Les entretiens ont été analysés à travers un processus de codage. Les objectifs étaient de comprendre: Comment ces acteurs ruraux ont vécu la pandémie et comment celle-ci les a impactés ? Comment ontils adapté leurs pratiques agricoles? Comment l'action communautaire s'est (re)déployée et quelles nouvelles solidarités ont émergé au sein des communautés oasiennes?

\section{Impacts de la pandémie sur différents acteurs dans la société oasienne}

Dans les deux vallées, la pandémie et le confinement ont eu de nombreux impacts sur le vécu des catégories les plus 
vulnérables (jeunes, femmes et petits agriculteurs), affectant ainsi leurs moyens de subsistance, comme nous l'illustrons dans ce qui suit.

\subsection{Une difficile commercialisation des produits agricoles}

La dépendance aux marchés nationaux et internationaux a contribué à la vulnérabilité des agriculteurs dans les deux contextes. Dans le Drâa, la récolte des pastèques a commencé en avril, quelques semaines après l'instauration du confinement. Cette culture attire des jeunes locaux qui exploitent des terres collectives. Ils se considèrent comme de petits agriculteurs et cultivent, sur quelques hectares, des pastèques destinées au marché et des cultures vivrières destinées à sécuriser la consommation familiale. En 2020, les jeunes ont éprouvé des difficultés à vendre leur production à des prix rémunérateurs, car seuls quelques acheteurs et intermédiaires ont pu accéder à la région. Les restrictions de mobilité et la difficulté d'obtenir une autorisation de déplacement a en effet rendu difficile l'accès à la vallée. Comme la pastèque ne se conserve pas, les jeunes ont été contraints d'accepter, à contrecœur, les prix offerts par les acheteurs. En outre, en temps normal, ceux-ci choisissaient les meilleures pastèques pour les exporter et les agriculteurs écoulaient les pastèques restantes dans les marchés locaux. Cependant, la fermeture des marchés les a poussés à laisser ces pastèques pourrir sur place ou à les donner au bétail.

Ce problème de commercialisation a concerné aussi les dattes. Dans le M'zab, la variété Deglet Nour est généralement stockée dans des chambres froides en attendant de trouver des marchés propices. La quasi-totalité de la production s'écoule habituellement à l'échelle nationale, et $5 \%$ de la production est exportée, principalement vers l'Europe (Bessaoud et al., 2019). Toutefois, suite à la fermeture des marchés et des frontières, à la suspension des moyens de transport publics et privés et aux interdictions de rassemblements familiaux, une grande partie de la production dattière de l'automne 2019 n'a pas été vendue. Ainsi, à l'automne 2020, les stations de refroidissement de la région étaient saturées, alors que les dattes de la nouvelle saison devaient être récoltées. En conséquence, une grande quantité de dattes stockées a dû être vendue à moitié prix ( \pm 200 dinars algériens $/ \mathrm{kg}$, soit $\pm 1,25 €)$ à des entreprises de transformation alimentaire pour libérer de la place pour la nouvelle production, alors qu'habituellement seules les dattes déclassées étaient destinées à la transformation. En outre, craignant de ne pas pouvoir vendre leur production et/ou de la vendre à perte, certains agriculteurs hésitaient à récolter les variétés commerciales, comme l'explique un agriculteur: «Le manque de visibilité du marché nous oblige à ne récolter que les dattes des variétés communes destinées à l'autoconsommation».

La volatilité du marché a affecté davantage les petits agriculteurs qui ne possèdent que quelques palmiers dattiers. Leur faible production fait qu'ils ne stockent pas leurs dattes et ont donc moins de marge de négociation des prix auprès des acheteurs. Dans le M'zab, à cause de l'incertitude du marché, peu d'acheteurs ont pris le risque d'acheter des dattes, poussant ainsi les agriculteurs qui avaient l'habitude de vendre leur production sur pied à ceux-ci, à laisser pourrir une partie de la production, perdant ainsi une partie de leur revenu.

\subsection{Une main-d'œuvre agricole rare et chère}

Dans les deux vallées, la récolte des dattes et des pastèques repose sur une main-d'œuvre régionale qui vient se joindre aux ouvriers locaux. Dans la vallée du Drâa, les moquefs, lieux où se réunissent les ouvriers à la recherche d'emploi journalier, ont été interdits, rendant ainsi leur recrutement difficile. La demande relativement élevée en main-d'œuvre par rapport à la disponibilité d'ouvriers agricoles qualifiés a entraîné une hausse des salaires. Par exemple, le prix du chargement d'un camion de pastèques, une tâche a priori anodine mais nécessitant une main-d'œuvre avisée, est généralement de 1400 à 1500 dirhams marocains $( \pm 130- \pm 140 €)$ alors qu'en 2020 , le prix a atteint $2000-2500 \mathrm{Dh}( \pm 185- \pm 230 €)$. Il en fut de même dans le M'zab où la faible disponibilité de la maind'œuvre qualifiée à l'échelle locale et l'incapacité des ouvriers originaires de Timimoun, située à $600 \mathrm{~km}$ de Ghardaïa, à se déplacer, ont entrainé une hausse des salaires, qui sont passés de 400 à $600 \mathrm{DA} /$ palmier $( \pm 2,50- \pm 3,75 €)$.

\subsection{La pluriactivité des familles oasiennes mise à l'épreuve}

Dans les oasis, les membres des familles s'engagent souvent dans des activités non-agricoles pour diversifier leurs revenus, notamment le tourisme, l'artisanat et les travaux de construction. Cette pluriactivité est essentielle pour faire face aux nouveaux défis, comme la difficulté de commercialisation des dattes. Toutefois, la pandémie a mis à rude épreuve cette pluriactivité et donc la capacité des familles oasiennes à répondre aux nouveaux défis, les rendant plus vulnérables. De plus, les jeunes qui travaillaient dans les grandes villes ont perdu leur emploi et sont rentrés se confiner avec leurs familles; cette mobilité est pourtant une caractéristique intrinsèque des jeunes pluriactifs, dont le travail agricole ne suffit pas à faire vivre le ménage (Ftouhi et al., 2015).

Dans les deux pays, les pouvoirs publics ont mis en place des initiatives pour soutenir les populations vulnérables. En Algérie, un montant de $10000 \mathrm{DA}( \pm 63 €)$ a été octroyé aux familles affectées par la pandémie et le confinement. Au Maroc, l'opération Tadamon, consistant à verser des indemnités pendant trois mois aux chefs de ménages opérant dans le secteur informel ou ayant perdu leur emploi, a été lancée. Toutefois, certaines familles affectées n'ont pas pu en bénéficier.

De plus, le secteur touristique a beaucoup souffert de la pandémie. Dans le Drâa, les personnes qui y travaillaient ont perdu leur emploi et se sont tournées vers l'aide de l'État. C'est le cas de Said, agent de sécurité dans un hôtel, qui, depuis l'instauration du confinement, vit de cette aide avec sa femme et ses enfants. Dans le M'zab, l'arrêt de l'activité touristique a affecté les artisans, comme l'explique un guide touristique: «La crise sanitaire a tué le tourisme. Les deux vagues de la pandémie [...] ont coïncidé avec la haute saison. Les artisans ont l'habitude de vendre de grandes quantités des produits locaux durant la fête du tapis organisée annuellement au printemps».

Les femmes oasiennes actives ont aussi vu leurs revenus, déjà modestes, diminuer. C'est le cas des adhérentes d'une coopérative agricole féminine du Drâa, spécialisée dans la fabrication du pain. En temps normal, elles vendaient 100 à 


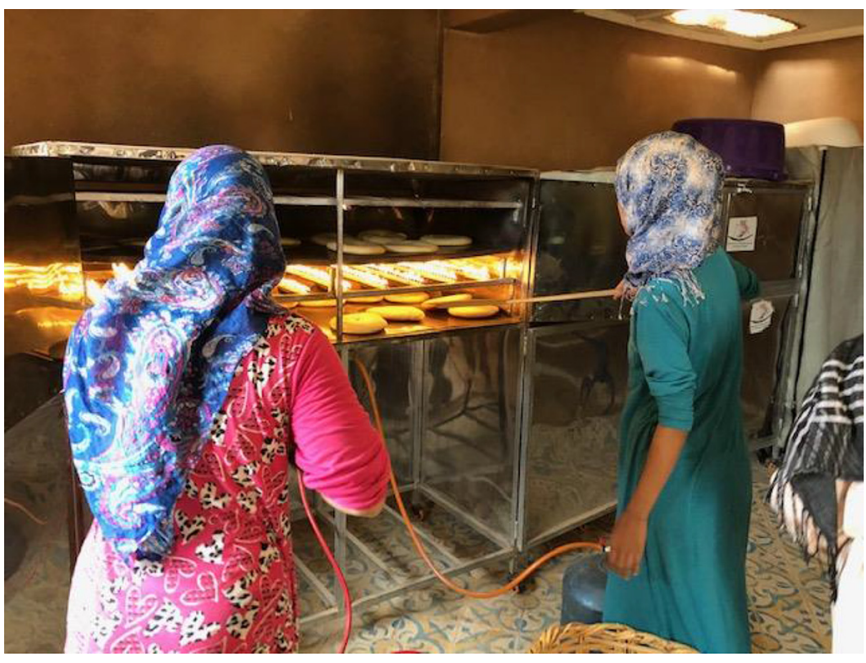

Fig. 2. Les membres d'une coopérative féminine dans la vallée du Drâa préparent du pain.

Fig. 2. Members of a feminine cooperative in the Draa Valley prepare bread.

200 pains par jour. Lors des fêtes et des funérailles, elles pouvaient vendre jusqu'à 600 pains par jour (Fig. 2). Mais en 2020, la vente n'a pas dépassé 20 pains par jour. Même si certaines adhérentes ont bénéficié des aides de l'État ou d'une ONG nationale, l'avenir de la coopérative reste incertain. Il en est de même pour les femmes du M'zab qui sont embauchées par les agriculteurs pour trier les dattes. Elles ont l'habitude de faire ce travail chez elles durant les heures où les enfants sont à l'école. Cependant, la difficulté d'écouler les dattes de la campagne 2019 a dissuadé les agriculteurs de faire appel à elles en 2020 .

\section{Réinventer les pratiques agricoles à l'ère de la Covid-19}

Nos entretiens illustrent non seulement comment la pandémie a affecté les moyens de subsistance des acteurs ruraux en les rendant encore plus vulnérables, mais aussi comment ils ont fait face à la situation. Dans ces différentes formes d'adaptation, la mobilisation du savoir-faire, des expériences antérieures et des réseaux sociaux sont essentiels en termes de résilience.

\subsection{Marché du travail virtuel}

Pour faire face au manque de main-d'œuvre, les jeunes producteurs de pastèques dans la vallée du Drâa ont mobilisé des groupes Facebook et WhatsApp pour recruter des ouvriers locaux, comme l'explique un ouvrier: "Vous donnez votre numéro de téléphone à un agriculteur et vous lui demandez de le partager dans son groupe. Lorsque quelqu'un a besoin d'ouvriers, il vous contacte... ». Lorsque les ouvriers n'avaient pas accès aux réseaux sociaux, ils utilisaient les informations fournies par leurs voisins ou amis possédant un smartphone, ou par le bouche-à-oreille.

\subsection{Des pratiques agroécologiques pour réduire les coûts}

Nos données montrent également des changements dans les pratiques agricoles. Dans le Drâa, pour minimiser les pertes, les jeunes producteurs de pastèques ont adapté leurs pratiques en s'appuyant sur leurs expériences agricoles. D'ailleurs, ils n'en étaient pas à leur première crise : l'année 2019 avait été désastreuse pour beaucoup d'entre eux car l'offre de pastèques dépassait la demande et de nombreux agriculteurs s'étaient retrouvés endettés. Par conséquent, certains ont minimisé les coûts en réduisant les intrants agricoles ou la surface cultivée en pastèques, en réutilisant les gaines de goutte-à-goutte, ou se sont tournés vers la diversification de leurs cultures. Ainsi, bien que la plupart d'entre eux n'aient réalisé que peu ou pas de bénéfices en 2020, ils ne se sont pas chargés de dettes supplémentaires. Dans le M'zab, les petits agriculteurs des oasis se sont tournés vers des pratiques puisées dans le répertoire agroécologique séculaire des oasiens, telles que l'utilisation du fumier et du jus de fumier comme engrais organiques et celle des semences locales. Par ailleurs, la forte demande locale en fumier a conduit à l'émergence d'un marché où les agriculteurs vendaient leur fumier, générant ainsi un revenu supplémentaire.

\subsection{Réinventer les solidarités et l'action collective}

Les pratiques de résilience ne se manifestent pas uniquement à travers des efforts individuels, mais aussi à travers des efforts collectifs. Par exemple, dans les oasis du M'zab, les personnes ayant émigré vers les villes sont revenues dans leur village natal lors de l'arrêt des activités économiques ou de leur scolarité. L'augmentation de la population s'ajoutant à l'essor des activités agricoles a accentué la pression sur les ressources en eau souterraine dans la vallée. Ceci a poussé les habitants de l'oasis de Ben Isguen à lancer une campagne de restauration du système d'irrigation communautaire comme l'explique le président d'une association locale: «La crise sanitaire nous a donné l'occasion de prendre soin de notre système d'irrigation communautaire». Cette initiative a été organisée par le biais de la touiza, un système traditionnel d'aide mutuelle qui caractérise la plupart des initiatives communautaires dans la vallée. Elle a permis de restaurer vingt-deux puits de recharge et "le niveau de la nappe phréatique dans les puits a augmenté de $5 \mathrm{~m}$ » selon un puisatier local. La pratique de la touiza a été également réinventé dans certains Ksour du Drâa où, en manque de maind'œuvre, les agriculteurs ont mobilisé des ouvriers locaux pour travailler à tour de rôle leurs terres, diminuant ainsi leurs charges (Fig. 3).

D'autres actions collectives ont été menées dans les deux contextes. Dans la vallée du M'zab, les femmes habituellement actives dans le tissage ont, avec l'appui d'associations locales, réorienté leur activité vers la fabrication des masques de protection. Plusieurs associations locales ont également apporté leur soutien aux jeunes subsahariens travaillant en tant qu'ouvriers agricoles, et une campagne de distribution de paniers de nourriture a été organisée pendant le mois de Ramadan. Dans certains Ksour de la vallée du Drâa, les jeunes 


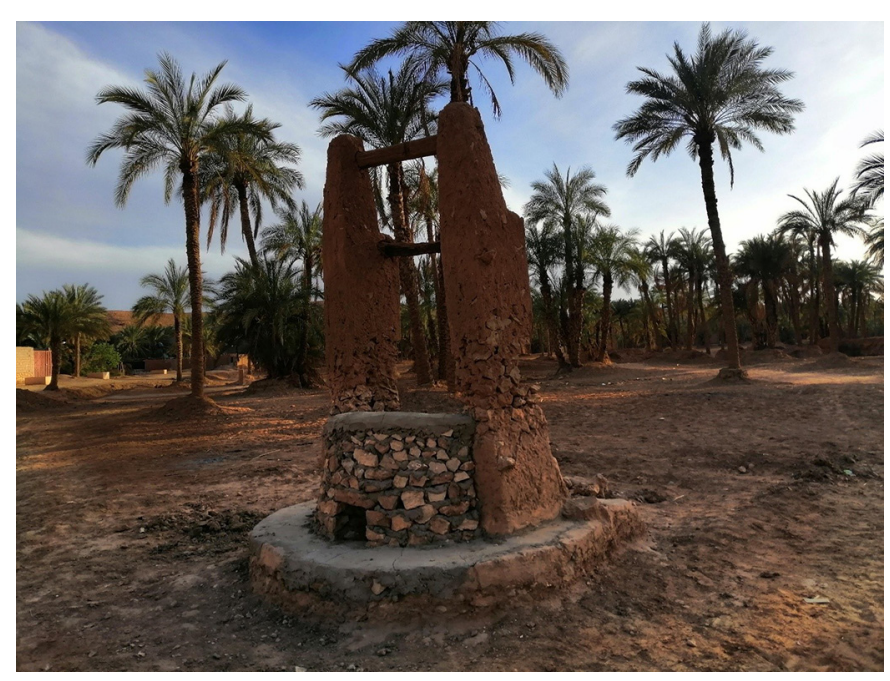

Fig. 3. Un puits de recharge réhabilité dans la vallée du M'zab. Fig. 3. A recharge well rehabilitated in the M'zab Valley.

ont organisé une campagne de collecte de fonds à travers un groupe WhatsApp, pour aider les familles dans le besoin et les agriculteurs dont les puits se sont taris, affectant ainsi leurs activités agricoles.

\section{Discussion et conclusion}

Dans cet article, nous avons montré comment différents acteurs ruraux de deux régions oasiennes du Maghreb ont fait face aux défis liés à la pandémie. Notre analyse a été menée auprès des catégories les plus vulnérables : petits agriculteurs, femmes et jeunes oasiens. Cette vulnérabilité s'explique en partie par la nature fragile des territoires oasiens en termes de ressources naturelles et de manque d'opportunités socioéconomiques, ce que la crise sanitaire a accentué.

Par ailleurs, dans les deux pays, les gouvernements ont soutenu leur discours public visant à rassurer les consommateurs «urbains» sur la sécurisation de l'alimentation en mettant en place certaines mesures. La fonction de l'agriculture est devenue celle de «nourrir le pays», sans s'interroger sur l'effet de ces mesures en termes de renforcement des inégalités. En effet, les marchés de gros, non concernés par la fermeture liée à la pandémie, et la connexion à l'export ont favorisé les grands agriculteurs et ceux ayant des moyens logistiques conséquents. Il en a été de même en Tunisie où ce sont les petits agriculteurs qui ont le plus souffert de la pandémie (Elloumi, 2020).

Le confinement imposé en Algérie et au Maroc a eu des impacts profonds sur la vie des populations rurales. Ceci s'est manifesté par la difficulté de commercialiser les produits agricoles et par une réduction de la pluriactivité, pourtant très importante pour certaines familles oasiennes. Malgré ces difficultés, les acteurs ruraux se sont engagés dans des pratiques résilientes, notamment à travers la mise en place d'une plateforme de travailleurs agricoles - un mouqef virtuel, la transition vers des pratiques agroécologiques et la commercialisation du fumier local. Les jeunes producteurs ont essayé d'être moins dépendants au marché, alors que d'autres ont minimisé les risques d'investissement et ont (ré) utilisé les ressources disponibles localement. On a également observé des pratiques collectives de résilience consistant à réactualiser des mécanismes de solidarité communautaires comme la touiza.

Certaines de ces pratiques de résilience sont intéressantes en termes de réhabilitation de la nature ou de reconnexion avec celle-ci. La restauration du système d'irrigation communautaire dans une oasis du M'zab l'illustre bien. En outre, dans la vallée du Drâa, la réduction des quantités d'intrants agricoles et la réutilisation des gaines de goutte-à-goutte, même si elle était dictée par des objectifs économiques, montre un potentiel important pour conserver la nature et pourrait servir de source d'inspiration pour d'autres agriculteurs.

Toutefois, ces pratiques résilientes ne signifient pas que l'État peut se déresponsabiliser au motif que les acteurs ruraux arrivent à se débrouiller seuls. Au contraire, il est impératif de se demander qui doit être résilient et pourquoi, et de remettre en cause la nature de la crise ainsi que les inégalités qu'elle a produites. Les pratiques de résilience, quant à elles, peuvent être mobilisées pour repenser les programmes d'appui (gouvernementaux et non-gouvernementaux) et pour en mettre en place de nouveaux, plus adaptés aux besoins et attentes des acteurs ruraux.

En conséquence, en accord avec d'autres travaux (Cote et Nightingale, 2012 ; Leonardelli et al., 2021), nous soutenons la nécessité de mobiliser le concept de résilience avec précaution. En effet, ce concept est souvent dépolitisé et mobilisé sans remettre en cause les origines de la crise et ses dimensions de pouvoir (Ibidem). Il devient ainsi un outil au service de programmes de gouvernance néolibérale, où l'État est déresponsabilisé et où les individus sont censés se transformer et tenir bon malgré les chocs exogènes et internes (Welsh, 2014), en comptant seulement sur leurs propres moyens et leurs initiatives. D'ailleurs, dans les deux cas étudiés, comme ailleurs dans le monde, la crise a interpellé les citoyens sur le rôle que devrait jouer l'État. En cela, il s'agit d'une reconnaissance du nécessaire rôle de l'État envers les sociétés, oasiennes en particulier. Toutefois, dans un pays comme le Maroc, où l'État a toujours associé plusieurs façons de gouverner, y compris en étant omniprésent (Hibou et Tozy, 2020), il est encore trop tôt pour tirer des enseignements sur un éventuel recul de la gouvernance néolibérale, puisque les initiatives lancées par l'État pour soutenir les populations affectées par la pandémie et le confinement sont ponctuelles et ne sont pas adaptées à toutes les catégories sociales.

\section{Références}

Amichi H, Kadiri Z, Bouarfa S, Kuper M. 2015. Une génération en quête d'opportunités et de reconnaissance : les jeunes ruraux et leurs trajectoires innovantes dans l'agriculture irriguée au Maghreb. Cahiers Agricultures 24: 323-329. https://doi.org/ 10.1684/agr.2015.0791.

Adger WN. 2000. Social and ecological resilience: are they related? Progress in Human Geography 24: 347-364. https://doi.org/ 10.1191/030913200701540465.

Banque Mondiale. 2019. Données sur les population rurale. https:// donnees.banquemondiale.org/indicator/SP.RUR.TOTL.ZS.

Bessaoud O, Pellissier JP, Rolland JP, Khechimi W. 2019. Rapport de synthèse sur l'agriculture en Algérie. Montpellier (France) : CIHEAM-IAMM, $82 \mathrm{p}$. 
Bossenbroek L, Ftouhi H. 2021. The plight of female agricultural wage workers in Morocco in times of the Covid-19 pandemic. Cahiers Agricultures 30: accepted.

Cote M, Nightingale AJ. 2012. Resilience thinking meets social theory: situating social change in socio-ecological systems (SES) research. Progress in Human Geography 36: 475-489. https://doi. org/10.1177/0309132511425708.

Elloumi M. 2020. L'agriculture tunisienne face à la Covid-19: impacts de la crise sanitaire et perspectives pour une agriculture résiliente. Cahiers Agricultures 29: 35. https://doi.org/10.1051/ cagri/2020032.

Ftouhi H, Kadiri Z, El Hassane A, Bossenbroek L. 2015. Partir et revenir au village. Mobilité non permanente des jeunes ruraux dans la région du Saïss (Maroc). Cahiers Agricultures 24: 372-378. https://doi.org/10.1684/agr.2015.0780.

Hamamouche MF, Saidani MA. 2020. Les oasis du M'zab au temps de la pandémie: adaptation de la population, émergence de nouvelles opportunités et renforcement des actions collectives et de solidarité. https://nessahra.net/les-oasis-du-mzab-au-temps-de-lapandemie-de-la-covid-19.

Hibou B, Tozy M. 2020. Tisser le temps politique au Maroc, imaginaire de l'État à l'âge néolibéral. Paris (France) : Les Éditions Karthala, 660 p.
Kadiri Z. 2020. Les Aït Débrouille. Rabat (Maroc) : Les Working Paper de la Chaire Fatéma Mernissi et du HEM Research Center, 14 p.

Keck M, Sakdapolrak P. 2013. What is social resilience? Lessons learned and ways forward. Erdkunde 76: 5-19. https://doi.org/ 10.3112/erdkunde.2013.01.02.

Lallau B. 2019. Développement : repolitiser la notion de résilience. Revue Projet 371: 74-78. https://doi.org/10.3917/pro.371.0074.

Lallau B, Laissus-Benoist P, Mbetid-Bessane E. 2018. Introduction : la résilience peut-elle passer de la théorie aux pratiques? Revue internationale des études du développement 3: 9-25. https://doi. org/10.3917/ried.235.0009.

Leonardelli I, Bossenbroek L, Ftouhi H, Kadiri Z, Bhat S, Kulkarni S, et al. 2021. Covid-19 in rural India, Algeria and Morocco: a feminist analysis of small-scale farmers' and agricultural laborers' experiences and inventive practices. Frontiers in Human Dynamics 3: 17. https://doi.org/10.3389/fhumd.2021.653979.

Mahdi M. 2020. Le douar de Tigouliane au croisement du Covid-19 et de la sécheresse (1/4). https://www.medias24.com/recit-le-douarde-tigouliane-au-croisement-du-covid-19-et-de-la-secheresse1-4-13598.html.

Welsh M. 2014. Resilience and responsibility: governing uncertainty in a complex world. The Geographical Journal 180: 15-26. https:// doi.org/10.1111/geoj.12012.

Citation de l'article : Ftouhi H, Saidani MA, Bossenbroek L, Hamamouche MF, Kadiri Z. 2021. Entre vulnérabilité et résilience : le vécu de la pandémie de Covid-19 dans deux sociétés oasiennes du Maghreb. Cah. Agric. 30: 30. 